\title{
Préparation du Canada en cas de grippe pandémique : Stratégie de laboratoire
}

\author{
B Henry ${ }^{1,2}$ au nom du Groupe de travail sur la préparation du Canada en cas de grippe \\ pandémique (GTPCP)*
}

\section{Résumé}

Le guide Préparation du Canada en cas de grippe pandémique : Guide de planification pour le secteur de la santé (PCGP) est un document d'orientation qui présente les activités de préparation clés du secteur de la santé conçues pour garantir que le Canada est prêt à réagir à la prochaine pandémie de grippe. Le présent article esquisse la stratégie de laboratoire du Canada en cas de grippe pandémique décrite dans l'Annexe à l'intention des laboratoires du PCGP. L'identification et la caractérisation en laboratoire d'un virus de la grippe pandémique sont essentielles pour détecter une pandémie, mettre au point un vaccin, déterminer la résistance aux antiviraux et éclairer les fonctions de surveillance, comme la surveillance de la propagation géographique de la maladie. Les principaux éléments de l'intervention incluent notamment de s'assurer qu'il y aura suffisamment de ressources pour réaliser toutes les activités. Les activités de la phase pré-analytique englobent notamment la réalisation adéquate de la collecte, du transport jusqu'au laboratoire, du triage et de la préparation des échantillons. Les activités analytiques renvoient aux différentes méthodes d'analyse pour la détection de la grippe, incluant le maintien de la capacité de culture du virus de la grippe pour la caractérisation génétique et antigénique. Dans la phase post-analytique, l'une des activités consiste à veiller à ce que les laboratoires de première ligne coordonnent leurs activités avec celles des laboratoires de santé publique provinciaux afin que les données et les échantillons soient accessibles aux fins de surveillance. Durant la période interpandémique, il est important de mettre en place les infrastructures, les protocoles et les processus qui permettront de lancer rapidement des recherches pendant la pandémie. Cette stratégie est un document évolutif qui sera régulièrement mis à jour.

\author{
Affiliations \\ 1 Présidente du GTPCP \\ 2 Bureau de l'administrateur \\ provincial de la santé, Victoria \\ (Colombie-Britannique)
}

\author{
${ }^{\star}$ Correspondance : \\ CPIPTGSecretariat- \\ GTPCPSecretariat@phac-aspc. \\ gc.ca
}

Citation proposée : Henry B au nom du Groupe de travail sur la préparation du Canada en cas de grippe pandémique (GTPCP). Préparation du Canada en cas de grippe pandémique : Stratégie de laboratoire. Relevé des maladies transmissibles au Canada. 2018;44(1):11-5. https://doi.org/10.14745/ccdr.v44i01a03f

\section{Introduction}

La capacité de détecter une pandémie de grippe, de mettre au point un vaccin pour protéger la population et réduire la propagation de la pandémie, ainsi que de détecter une résistance aux antiviraux qui limiterait l'efficacité de la réserve canadienne d'antiviraux, dépend de l'identification et de la caractérisation du nouveau virus en question. Les laboratoires assument cette fonction en menant des analyses conçues pour distinguer une nouvelle souche de grippe de celles de la grippe saisonnière ou d'autres virus des voies respiratoires. Ces données de laboratoire sont utilisées pour éclairer les fonctions de surveillance telles que la surveillance de la propagation géographique de la maladie et les répercussions des interventions.

La stratégie de laboratoire du Canada en cas de grippe pandémique est décrite dans l'Annexe à l'intention des laboratoires (1) du document Préparation du Canada en cas de grippe pandémique: Guide de planification pour le secteur de la santé (PCGP) (2). Cette annexe s'appuie sur l'expérience que les laboratoires ont acquise lors de la pandémie de grippe H1N1 de 2009, qui a mis en évidence l'importance d'une communication et d'une coordination efficaces entre tous les paliers de laboratoires et leurs contreparties tout au long de l'intervention en cas de pandémie. Ce document d'orientation technique décrit une approche extensible pour la prestation des services de laboratoire pendant une pandémie, accompagnée d'éléments déclencheurs des interventions et d'autres outils qui offrent la souplesse nécessaire pour adapter les activités des laboratoires à l'accroissement et à la variabilité de la demande en analyses. Il cible les professionnels des laboratoires cliniques nationaux, provinciaux et hospitaliers, les cliniciens, les épidémiologistes et les autres intervenants ayant des responsabilités qui recoupent celles de ces laboratoires, ainsi que les autres personnes intéressées. Le présent article propose un résumé de l'Annexe à l'intention des laboratoires (1) du PCGP, qui a été mise à jour récemment. Ce numéro du Relevé des maladies transmissibles au Canada (RMTC) $(3,4)$ contient également d'autres résumés sur la stratégie de surveillance et les directives de planification à l'intention du secteur de la santé. 


\section{Stratégie de laboratoire du Canada en cas de grippe pandémique}

\section{Objectifs}

Les objectifs des analyses en laboratoire du virus de la grippe au cours d'une pandémie sont regroupés en deux grands volets : la surveillance de la population et les épreuves diagnostiques. La surveillance de la population vise à détecter et à identifier le nouveau virus et à le distinguer des souches courantes, ce qui inclut la détermination de sa sensibilité aux antiviraux et la caractérisation de la souche, permettant ainsi de relever une éventuelle non-concordance avec les vaccins. Si les épreuves diagnostiques chez les patients présentant un syndrome grippal (SG) peuvent ne pas être indiquées pour la prise en charge clinique des personnes présentant un SG sans complications, les analyses joueront tout de même un rôle dans la surveillance communautaire des éclosions et dans le diagnostic en temps opportun des patients hospitalisés et présentant un risque élevé afin d'éclairer les décisions en matière de traitement et de prise en charge des contacts exposés.

\section{Contexte canadien}

Les laboratoires agréés pour mener les activités analytiques requises en cas de pandémie sont gérés par les autorités fédérales et provinciales. Le Laboratoire national de microbiologie (LNM), les laboratoires provinciaux de santé publique (LPSP) et les laboratoires de première ligne des centres hospitaliers font partie de cette catégorie. II est essentiel que les laboratoires de tous les échelons collaborent entre eux, en fonction de rôles clairement définis et de structures et processus adéquats, de façon à permettre la détermination et la prestation rapides des mesures d'intervention en santé publique au cours d'une pandémie.

L'Agence de la santé publique du Canada (ASPC), par l'entremise du LNM, est responsable de coordonner la surveillance en laboratoire à l'échelle nationale et, sur la scène internationale, de rendre compte des résultats de laboratoire à l'Organisation mondiale de la Santé et à ses partenaires. Le LNM joue un rôle important de soutien aux LPSP grâce à des fonctions de laboratoire précises, comme la caractérisation génétique et antigénique des souches de la grippe saisonnière et des nouvelles souches grippales, l'analyse de résistance phénotypique aux antiviraux, de même que la diffusion de I'information et le soutien aux LPSP pour la mise au point et la validation d'épreuves diagnostiques pour les nouvelles souches en vue d'en décentraliser I'utilisation. Les LPSP utilisent les épreuves de détection primaires et ont la responsabilité de s'assurer de disposer de la capacité de détecter l'émergence d'un nouveau sous-type viral potentiel. Ils jouent un rôle de soutien auprès des laboratoires de première ligne et fournissent au LNM, au moyen des systèmes de surveillance établis, des échantillons viraux, des échantillons prélevés chez des patients et des renseignements épidémiologiques limités. Parmi les responsabilités des laboratoires de première ligne en cas de pandémie, mentionnons les analyses pour détecter le virus de la grippe dans les échantillons prélevés chez des patients et l'envoi des échantillons de diagnostic aux LPSP aux fins de caractérisation plus poussée.
La collaboration est essentielle pour répondre à la demande et à la capacité d'analyse, qui varieront entre les différentes régions du pays. Compte tenu de la taille du Canada et de la distribution de sa population, une pandémie touchera probablement les différentes régions à des moments et à des degrés différents, et les laboratoires des régions les plus touchées seront donc confrontés à une demande plus grande en services d'analyse. La réalisation d'analyses est aussi plus difficile dans les villages éloignés et isolés, ce qui exige une collaboration accrue entre les différents territoires de compétence. Les laboratoires de la Colombie-Britannique et de I'Alberta, par exemple, effectuent les analyses pour les territoires, ce qui nécessite une bonne préparation sur le plan de la logistique pour la collecte et le transport des échantillons.

\section{Éléments clés de l'intervention des laboratoires}

\section{Activités pré-analytiques}

Les activités pré-analytiques sont celles qui doivent être réalisées pour garantir la collecte adéquate des échantillons et leur transport vers le laboratoire aux fins d'analyse. On a souvent recours à différents types d'échantillons et de méthodes de collecte pour optimiser la détection du virus de la grippe chez les patients atteints d'une forme grave de la maladie. Les conditions et le moment du transport sont des facteurs importants dont il faut tenir compte pour assurer l'intégrité des échantillons.

Au cours de la pandémie de 2009, de nombreux laboratoires ont sous-estimé les pressions associées à l'augmentation de la demande d'analyses sur le processus pré-analytique. II faut adopter des stratégies qui garantiront que les ressources disponibles suffiront à cette demande, comme augmenter les ressources pour l'enregistrement des échantillons reçus au laboratoire (p. ex., pour la réception, le tri, la saisie dans le système d'information du laboratoire, l'étiquetage et le traitement). Un processus devrait être mis en place au préalable pour effectuer le triage des échantillons durant les périodes d'activité accrue. Les laboratoires devraient aussi élaborer un processus pour aliquoter (c'est-à-dire diviser ou répartir) les échantillons de façon à permettre d'analyser de nouveau un prélèvement ou d'en remettre un échantillon au LNM, au besoin.

\section{Activités analytiques}

Il existe plusieurs méthodes différentes pour la détection du virus de la grippe, chacune avec ses propres caractéristiques : délai pour l'obtention de résultats, sensibilités, capacité de caractérisation des sous-types, débit et coûts. Les analyses les plus recommandées pour la détection et la caractérisation de la grippe sont les méthodes de test d'amplification des acides nucléiques (TAAN) en raison de leur rendement, de la possibilité de les automatiser et de leur extensibilité. Les épreuves d'immunofluorescence directe et les épreuves d'immunofluorescence indirecte peuvent servir à détecter le virus de la grippe $A$, mais ne permettent pas le sous-typage et sont moins sensibles que les méthodes de TAAN. Les tests de diagnostic rapide de la grippe (TDRG), qui s'appuient sur la détection d'antigènes, permettent d'obtenir des résultats en 30 minutes, mais ils ne permettent pas le sous-typage et 
leur faible sensibilité limite leur utilité pour la prise en charge individuelle des patients. Les TDRG peuvent toutefois contribuer à la surveillance des éclosions ou être une option pour détecter rapidement la présence du virus de la grippe dans les villages éloignés. Les utilisateurs finaux doivent bien comprendre les limites des TDRG s'appuyant sur la détection d'antigènes avant de les employer. Des TAAN plus rapides font leur apparition, mais leur rendement pour la détection de nouveaux virus et leur incidence sur les résultats pour les patients doivent être étudiés plus en profondeur. Les tests sérologiques exigent beaucoup de travail et ne sont pas couramment utilisés pour le diagnostic, mais ils se sont avérés utiles dans le cadre d'études épidémiologiques et immunologiques.

Il est important de maintenir une capacité de culture du virus de la grippe, car les isolats viraux sont indispensables à la caractérisation génétique et antigénique, à la surveillance des dérives antigéniques et à l'analyse de la résistance phénotypique aux antiviraux. On s'attend toutefois à ce que les nouveaux virus de la grippe soient considérés comme des agents pathogènes du groupe de risque 3 , ce qui fait que seuls les LPSP possédant un permis de niveau de confinement 3 peuvent réaliser cette activité. Les travaux continus de caractérisation génétique et antigénique et d'analyse de la résistance aux antiviraux constituent des aspects importants de la surveillance courante. En outre, les analyses de résistance phénotypique et génotypique aux antiviraux sont réalisées dans le cadre d'analyses ciblées d'échantillons prélevés chez des patients soupçonnés d'être infectés par un virus résistant. Les analyses de résistance aux antiviraux éclairent les lignes directrices sur I'utilisation des antiviraux et peuvent représenter un complément important en vue de la prise en charge clinique des patients sur une base individuelle.

Lors d'une pandémie de grippe, d'autres virus des voies respiratoires (comme le parainfluenza et le rhinovirus) peuvent circuler dans la population et causer d'importantes maladies. Pour veiller à ce que la morbidité et la mortalité soient correctement attribuées à la grippe pandémique, il est important de maintenir une certaine capacité d'analyse des autres virus respiratoires, même lorsque les ressources deviennent limitées.

\section{Activités post-analytiques}

Il est important de veiller à ce que les laboratoires de première ligne coordonnent leurs activités avec celles des LPSP afin que les données et les échantillons soient accessibles aux fins de surveillance. Si une demande accrue en analyses exige que l'on modifie les méthodes d'analyse en laboratoire, ces changements doivent être communiqués aux cliniciens et aux autres utilisateurs des données de laboratoire et il faut également signaler clairement les répercussions sur la surveillance ou les soins aux patients. II faudrait préparer une stratégie de communication pendant la période de grippe saisonnière, de façon à garantir qu'un processus et des infrastructures sont en place pour rédiger et diffuser les messages pendant une pandémie. Les laboratoires doivent aussi planifier l'archivage, l'entreposage et le retrait des grandes quantités d'échantillons qui seront traités pendant une pandémie.

\section{Assurance de la qualité et contrôle de la qualité}

Tous les laboratoires qui effectuent des épreuves diagnostiques de la grippe doivent participer aux programmes de vérification de la compétence en matière de virus de la grippe, et les activités de contrôle de la qualité devraient se poursuivre à mesure que la pandémie évolue. Le LNM offre des panels de vérification de la compétence pour évaluer le rendement des épreuves effectuées dans les LPSP et les autres laboratoires. II communique aussi aux LPSP les données sur la séquence pour assurer l'efficacité des analyses servant à identifier le nouveau sous-type. Si, comme lors de la pandémie de 2009, un nouveau virus nécessite de nouveaux protocoles d'analyse, les LPSP et le LNM travailleront de concert à la validation de l'exactitude des nouvelles méthodes ou des épreuves offertes sur le marché.

\section{Considérations en matière de biosécurité}

Les laboratoires doivent suivre les protocoles de biosécurité pour prévenir l'exposition en laboratoire à un nouveau virus au moment d'analyser les échantillons. Le Centre de la biosûreté de I'ASPC fournira des directives sur la façon de manipuler les échantillons d'un nouveau virus et ces directives seront mises à jour à mesure que de nouveaux renseignements seront disponibles (5).

\section{Intégration des fonctions de laboratoire aux autres éléments du PCGP}

Les laboratoires et les décideurs du secteur de la santé publique devraient travailler ensemble durant la période interpandémique pour veiller à ce que l'on connaisse et comprenne les fonctions de laboratoire, notamment en ce qui concerne les exigences particulières associées à la détection du virus de la grippe lors d'une pandémie et le rôle important des laboratoires dans I'intervention en cas de pandémie. En outre, le partage des données entre les laboratoires, les autorités provinciales et territoriales et l'ASPC pendant une pandémie est de la plus haute importance. Des ententes de partage des données devraient être conclues avant une pandémie pour faciliter le transfert de données; ces ententes doivent aborder les questions de la propriété intellectuelle, des droits d'auteur et d'autres questions touchant la publication.

Il existe plusieurs liens et interrelations clés avec les activités de laboratoire qui contribueront à une intervention coordonnée et efficace en cas de pandémie. Afin de s'assurer que les données sont comparables et interprétées correctement, les épidémiologistes doivent comprendre les détails des analyses effectuées en laboratoire (comme les algorithmes de détection, la sensibilité et la spécificité des épreuves effectuées, etc.). Il est tout aussi important que les laboratoires comprennent de quelles données les épidémiologistes ont besoin pour réaliser leurs évaluations des risques et analyser l'évolution de la pandémie. L'utilisation des infrastructures de surveillance déjà en place pour la grippe saisonnière et les autres virus des voies respiratoires, de même que la mise en place d'ententes de partage des données durant la période interpandémique, permettent d'optimiser la capacité de surveillance au cours d'une pandémie (6). Les laboratoires devraient communiquer aux cliniciens et autres 
utilisateurs finaux les changements apportés aux pratiques d'analyse en laboratoire, notamment les modifications aux exigences en matière de collecte et au rendement des épreuves, de façon à ce que les cliniciens comprennent comment ces changements pourraient toucher et limiter la prise en charge des patients. Les planificateurs communautaires doivent collaborer avec les experts de laboratoires et les provinces et territoires pour trouver de nouvelles façons d'offrir des services d'analyse aux Premières Nations et aux patients des villages éloignés et isolés, et de diffuser l'information entre les partenaires. L'emplacement géographique et les conditions météorologiques peuvent être des facteurs importants à considérer au moment de planifier le transport d'échantillons vers un laboratoire, étant donné que les échantillons sont sensibles aux délais aussi bien qu'à la température. Enfin, les laboratoires devraient établir les processus de communication nécessaires avec les fournisseurs pour être en mesure d'accéder rapidement aux stocks commerciaux d'épreuves et de réactifs pour soutenir l'intervention des laboratoires.

\section{Besoins en matière de recherche}

Durant la période interpandémique, il est important de mettre en place les infrastructures, les protocoles et les processus qui permettront de lancer rapidement des recherches pendant une pandémie afin d'aider à combler les lacunes dans les connaissances sur la prévention, le traitement et les stratégies de contrôle de la grippe. Compte tenu du rôle qu'ils jouent pour soutenir ces recherches, les laboratoires devraient participer à cette planification préalable. Ils devraient aussi planifier les infrastructures dont ils auraient besoin pour soutenir ces recherches. On devrait contribuer à la préparation de la recherche en menant rapidement des études sur la grippe saisonnière durant la période interpandémique.

\section{Discussion}

La stratégie de laboratoire du PCGP emploie les algorithmes de détection et les ententes de collaboration et de partage des données qui forment le système de surveillance et d'analyse de la grippe saisonnière, et cette stratégie a été mise à jour pour y intégrer les leçons retenues de la pandémie de grippe H1N1 de 2009. II reste toutefois des difficultés à surmonter, qui sont indiquées en tant que suggestions d'améliorations à apporter au processus de préparation que les laboratoires de tous les territoires de compétence devraient envisager durant les périodes interpandémiques.

L'une des principales difficultés est l'augmentation anticipée de la demande en analyses durant une pandémie, qui peut être plus que décuplée par rapport à la demande saisonnière. Des plans devraient être élaborés durant la période interpandémique pour gérer cette demande et inclure ceux qui concernent les fonctions opérationnelles telles que les politiques d'embauche et de formation du personnel pour répondre à la demande accrue, les facteurs à considérer pour le traitement de volumes élevés d'échantillons et les plans pour répondre à la demande en matière de fournitures de laboratoire. Les laboratoires de première ligne devraient mettre cette période à profit pour renforcer leur capacité de diagnostic, alors que les provinces et territoires devraient appliquer les critères prévus par les LPSP pour établir l'ordre de priorité des analyses, de façon à assurer l'uniformité des rapports à l'échelle nationale.

Les stratégies de communication pourraient aussi être renforcées durant la période interpandémique afin de faciliter l'échange des données en temps opportun, notamment pour ce qui est $d$ 'améliorer la coordination entre les LPSP et les laboratoires de première ligne pour la communication des données de surveillance. Tout au long des périodes interpandémiques, on devrait entretenir les liens au sein du Réseau des laboratoires de santé publique du Canada (RLSPC) (7) et des autres groupes similaires et soutenir la tenue de réunions régulières afin de maintenir l'efficacité continue du RLSPC dans la coordination à l'échelle nationale des analyses, comme il l'a fait durant la pandémie de 2009 (8).

Le RLSPC continue de surveiller les contributions des laboratoires aux processus de préparation et d'intervention en cas de grippe pandémique. En consultation avec le Réseau de préparation des laboratoires à une pandémie d'influenza, le RLSPC révise les protocoles des laboratoires pour veiller à ce que les laboratoires canadiens soient en mesure de détecter un nouveau virus de la grippe qui ferait son apparition au pays. Enfin, le RLSPC supervise également les révisions de l'Annexe à l'intention des laboratoires du PCGP et y intègre les faits nouveaux qui sont mis au jour.

\section{Conclusion}

Les analyses en laboratoire sont une fonction essentielle de l'intervention en cas de pandémie de grippe et elles contribuent à la fois aux travaux de surveillance épidémiologique et à la prise en charge clinique des personnes touchées. Elles profitent des systèmes et des structures qui sont utilisés et améliorés chaque année pour la grippe saisonnière et les autres virus des voies respiratoires, mais il faut anticiper les besoins accrus en cas de pandémie et accroître les activités pour répondre à ces besoins.

\section{Déclaration des auteurs}

Membres du Groupe de travail sur la préparation du Canada en cas de grippe pandémique (GTPCP) : Henry B. (présidente), Alfieri A., Gant S., Gemmill I., Hatchette T., Jayaraman G., Schwartz B.

Secrétariat du GTPCP : Paddle L., Stirling R., Gadient S.

ASPC : Charos G., Williams J.

LNM : Marcino D., Kushcak T.

\section{Conflit d'intérêt}

Aucun.

\section{Remerciements}

Merci à Anne Wiles, qui a préparé la première ébauche de ce résumé. 


\section{Financement}

Les travaux du GTPCP sont financés par l'Agence de la santé publique du Canada.

\section{Références}

1. Agence de la santé publique du Canada. Linges directrices à l'intention des laboratoires : Préparation du Canada en cas de grippe pandémique : Guide de plannification pour le secteur de la santé. Ottawa (ON): ASPC; 2015. https://www.canada.ca/fr/sante-publique/services/grippeinfluenza/preparation-canada-cas-grippe-pandemiqueguide-planification-secteur-sante/annexe-a-intention-deslaboratoires.html

2. Agence de la santé publique du Canada. Préparation du Canada en cas de grippe pandémique : Guide de planification pour le secteur de la santé. Ottawa (ON): ASPC; 2017. https://www.canada.ca/fr/sante-publique/ services/grippe-influenza/preparation-canada-cas-grippepandemique-guide-planification-secteur-sante.html

3. Henry B au nom du Groupe de travail sur la préparation du Canada en cas de grippe pandémique (GTPCGP). Préparation du Canada en cas de grippe pandémique : Guide de planification pour le secteur de la santé. Relevé des maladies transmissibles au Canada. 2018;44(1):7-10. https://www.canada.ca/fr/sante-publique/services/rapportspublications/releve-maladies-transmissibles-canada-rmtc/ numero-mensuel/2018-44/rmtc-volume-44-1-4-janvier-2018/ preparation-canada-cas-grippe-pandemique.html
4. Henry B au nom du Groupe de travail sur la préparation du Canada en cas de grippe pandémique (GTPCP). Préparation du Canada en cas de grippe pandémique : Stratégie de surveillance. Relevé des maladies transmissibles au Canada. 2018;44(1):16-20. https://www.canada.ca/fr/santepublique/services/rapports-publications/releve-maladiestransmissibles-canada-rmtc/numero-mensuel/2018-44/rmtcvolume-44-1-4-janvier-2018/preparation-canada-cas-grippepandemique-strategie-surveillance.html

5. Agence de la santé publique du Canada. À propos du Centre de la biosûreté. Ottawa (ON): ASPC; 2017. https:// www.canada.ca/fr/sante-publique/services/biosecuritebiosurete-laboratoire/a-propos-centre-biosurete.html

6. Agence de la santé publique du Canada. Surveillance : Préparation du Canada en cas de grippe pandémique : Guide de planification pour le secteur de la santé. Ottawa (ON): ASPC; 2015 Annexe traitant de la surveillance. https:// www.canada.ca/fr/sante-publique/services/grippe-influenza/ preparation-canada-cas-grippe-pandemique-guideplanification-secteur-sante/annexe-traitant-de-la-surveillance. html

7. Agence de la santé publique du Canada. le Réseau des laboratoires de santé publique du Canada (RLSPC). Ottawa (ON): ASPC; 2015. https://www.nml-Inm.gc.ca/cphln-rlspc/ index-fra.htm

8. Comité sénatorial permanent des affaires sociales, des sciences et de la technologie. La réponse du Canada à la Pandémie de grippe H1N1 de 2009. Ottawa (ON): Sénat du Canada; 2010. https://sencanada.ca/content/sen/ Committee/403/soci/rep/rep15dec10-f.pdf 